HEFAT2012

9th International Conference on Heat Transfer, Fluid Mechanics and Thermodynamics 16 - 18 July 2012

\title{
Natural convection and entropy generation in a large aspect ratio cavity with walls of finite thickness.
}

Malta

\author{
D. Pastrana, J. C. Cajas, C. Treviño ${ }^{2}$ \\ Facultad de Ciencias, Universidad Nacional Autónoma de México. \\ ${ }^{2}$ Facultad de Ciencias y UMDI Sisal, Yucatán, Universidad Nacional Autónoma de México. \\ E-mails: dpastrana@ciencias.unam.mx,jc.cajas@gmail.com,ctrev@gmail.com
}

\begin{abstract}
In this work, the heat transfer and fluid flow process in a vertical cavity of large aspect ratio, $A R=12$, with walls of finite thickness, heated from two portions localized in the side walls of the cavity near the bottom, is studied. The equations of motion are written in non-dimensional form, depending of five non dimensional parameters (the Rayleigh number, the Prandtl number, the thermal and conductivity ratio of the fluid and the material of the cavity and the non-dimensional width of the walls) and are solved numerically by the use of the SIMPLE algorithm. Calculations were performed for three different values of the Rayleigh number for two values of the thickness of the wall, and fixed values for the rest of parameters.
\end{abstract}

\section{Introduction}

In many of the practical systems where natural convection is present, it is of prime importance to consider the thermal properties of the material that confines the fluid. A deeper knowledge of the combined effects of the heat conduction in the walls of the cavity, and the natural convection in the fluid, can lead to an improvement in the design of thermal exchanging devices.

The conjugated effect of conduction and natural convection has been widely studied, some examples are cited next. Kim and Viskanta (1985) performed a numerical and experimental study of the natural convection in a square cavity made of four conductive walls. They found that under certain configurations of the system, the conductive walls help to partially stabilize the flow and reduce the temperature differences in the cavity, as well as the heat transfered by natural convection. More recently, Liaqat and Baytas (2001), Moghtada and Mobedi (2008) and Zhang et al. (2011) studied the influence of the presence of walls of finite thickness over the natural convection in square cavities with different geometrical configurations. The numerical results reveal that, among other things, the heat transfer increases almost linearly with the ratios of thermal conductivities or diffusivities and decreases if the cavity is inclined. Their results have demonstrated that there exists a significant change in the behavior of the resultant flow, in comparison with those obtained in similar systems where the conjugated phenomena is not considered.

In the last years, the heat transfer studies for the design of thermal devices have relied on the analyses of the second law of thermodynamics and on the minimal entropy production. However, there are few works that consider the entropy generation due to heat conduction and natural convection in enclosures with walls of finite thickness. The works performed by Varol et al. stand out (Varol et al. (2008) and Varol et al. (2009)) where the entropy production due to the conjugated process of heat conduction-natural convection is studied in a square cavity with side walls of different finite thicknesses and in a trapezoidal porous cavity with a solid vertical wall of finite thickness. Among their more important results, the presence of the solid walls affects the temperature and velocity fields inside the cavity, the Bejan number diminishes when the Rayleigh number or the thermal conductivities ratio increases, and the most intense zones of local entropy production are found in the corners of the cavity. They also show that the entropy produced by the viscous effects does not depend on the thickness of the walls, and that the shape of the cavity can be a controlling parameter to decrease the overall entropy production, obtaining an energy saving.

The present work is aimed to provide further information on the transient heating process and the entropy production in a large aspect ratio cavity with walls of finite thickness. Which is a system that can be used in many engineering devices.

\section{Problem statement}

In this work, the natural convection and entropy generation in a Boussinesq fluid with constant viscosity and thermal diffusivity, 
confined by a two dimensional rectangular cavity with large aspect ratio, $A R=L / H=12$, and with solid conductive walls of finite thickness with constant thermal diffusivity, as shown in Fig. 1, is studied. $L$ is the interior height of the cavity and $H$ is its interior length. The whole system, conformed by the cavity and the walls, is adiabatically isolated from the exterior, except by two portions of height $l=H$ and length $h$ located symmetrically on the side walls of the cavity at a distance $L_{1}$ from the top wall. The two portions are held at constant temperature $T_{1}$ which is larger than the initial temperature of the fluid $T_{0}$.

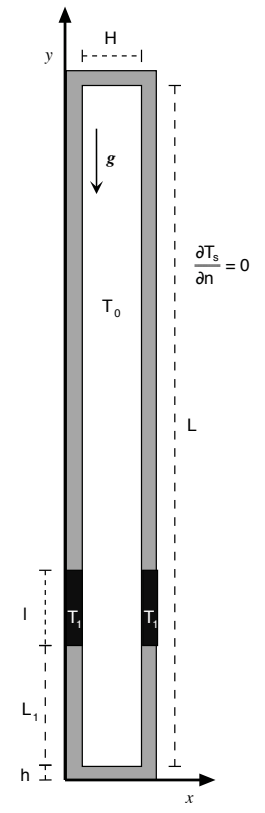

Fig. 1: Schematic representation of the cavity.

Using the length of the cavity $H$, the diffusion time of temperature $H^{2} / \kappa_{f}$ ( $\kappa_{f}$ is the thermal diffusivity of the fluid) and the difference between the initial temperature of the fluid and the temperature that induces the buoyancy forces $T_{1}-T_{0}$, the following non-dimensional variables arise,

$$
\begin{gathered}
x_{i}=\frac{x_{i}^{*}}{H}, \quad u_{i}=\frac{u_{i}^{*} H}{\kappa_{f}}, \quad t=\frac{t^{*} \kappa_{f}}{H^{2}}, \\
p=\frac{p^{*} H^{2}}{\rho_{0} \kappa_{f}^{2}}, \quad \theta_{f}=\frac{T_{f}-T_{0}}{T_{1}-T_{0}}, \quad \theta_{s}=\frac{T_{s}-T_{0}}{T_{1}-T_{0}} .
\end{gathered}
$$

where $x_{i}$ and $u_{i}$ are the non-dimensional $i$-th components of the position and velocity vectors respectively. $p, t$ and $\theta$ are used to denote the non-dimensional pressure, time and temperature repectively, the super index $*$ is used to distinguish between dimensional and non-dimensional quantities, and the sub indexes $f$ and $s$ distinguish the physical properties of the fluid from those of the solid,

With the use of the non-dimensional variables (1), the governing equations of the system can be written as follow

$$
\begin{array}{r}
\frac{\partial u}{\partial x}+\frac{\partial v}{\partial y}=0 ; \quad \frac{D u}{D t}=-\frac{\partial P}{\partial x}+\operatorname{Pr}\left(\frac{\partial^{2} u}{\partial x^{2}}+\frac{\partial^{2} u}{\partial y^{2}}\right), \\
\frac{D v}{D t}=-\frac{\partial P}{\partial y}+\operatorname{RaPr} \theta_{f}+\operatorname{Pr}\left(\frac{\partial^{2} v}{\partial x^{2}}+\frac{\partial^{2} v}{\partial y^{2}}\right), \\
\frac{D \theta_{f}}{D t}=\frac{\partial^{2} \theta_{f}}{\partial x^{2}}+\frac{\partial^{2} \theta_{f}}{\partial y^{2}} ; \quad \frac{\partial \theta_{s}}{\partial t}=\alpha\left(\frac{\partial^{2} \theta_{s}}{\partial x^{2}}+\frac{\partial^{2} \theta_{s}}{\partial y^{2}}\right),
\end{array}
$$

with the following boundary conditions

$u_{i}=0, \quad$ where $i=1,2, \quad \theta_{s}=\theta_{f}, \quad$ on the solid-fluid interface;

$$
K \frac{\partial \theta_{s}}{\partial n}=\frac{\partial \theta_{f}}{\partial n} \quad \text { on the solid-fluid interface; }
$$

$$
\begin{gathered}
\theta_{s}=1, \quad \text { en: } y \in\left[\frac{L_{1}+h}{H}, \frac{L_{1}+h+l}{H}\right], \quad x \in\left[0, \frac{h}{H}\right] \quad \text { and } \\
y \in\left[\frac{L_{1}+h}{H}, \frac{L_{1}+h+l}{H}\right], \quad x \in\left[\frac{h}{H}+1, \frac{2 h}{H}+1\right] \\
\frac{\partial \theta_{s}}{\partial n}=0 \quad \text { on the exterior part of the walls, }
\end{gathered}
$$

where the usual non-dimensional parameters have been defined, the Prandtl's number $\operatorname{Pr}=v / \kappa_{f}$, with $v$ as the kinematic viscosity; the Rayleigh's number $R a=\beta g d^{3}\left(T_{1}-T_{0}\right) /\left(v \kappa_{f}\right), \beta$ is the volumetric thermal expansion coefficient; the thermal diffusivities ratio is $K=k_{s} / k_{f}$ and $n$ represents the normal direction to a surface.

\subsection{Entropy Production}

In the Linear Irreversible Thermodynamics formulation, an explicit expression for the entropy balance is obtained in terms of the velocity and temperature fields. From that expression, the internal entropy production in terms of the non-dimensional variables are

$$
\begin{gathered}
\sigma_{q}^{*}=\sigma_{q} \frac{H^{2} T_{0}^{2}}{k\left(T_{1}-T_{0}\right)^{2}}=\frac{1}{(1+\varepsilon \theta)^{2}}\left[\left(\frac{\partial \theta}{\partial x}\right)^{2}+\left(\frac{\partial \theta}{\partial y}\right)^{2}\right] \\
\sigma_{v}^{*}=\sigma_{v} \frac{H^{2} T_{0}^{2}}{k\left(T_{1}-T_{0}\right)^{2}}=\frac{E c}{(1+\varepsilon \theta)}\left(\frac{\partial u}{\partial y}+\frac{\partial v}{\partial x}\right)^{2} .
\end{gathered}
$$

where $\sigma_{q}$ represents the entropy production due to heat flux, and $\sigma_{v}$ is the corresponding to the viscous effects inside the fluid. $E c$ is the Eckert number given by $E c=v \kappa /\left[2 c_{p}\left(T_{1}-T_{0}\right) H^{2}\right]$ and $\varepsilon=\left(T_{1}-T_{0}\right) / T_{0}$. In the present work $0<\varepsilon \ll 1$, the temperature differences are considered to be small in comparison with the tem- 
perature itself.

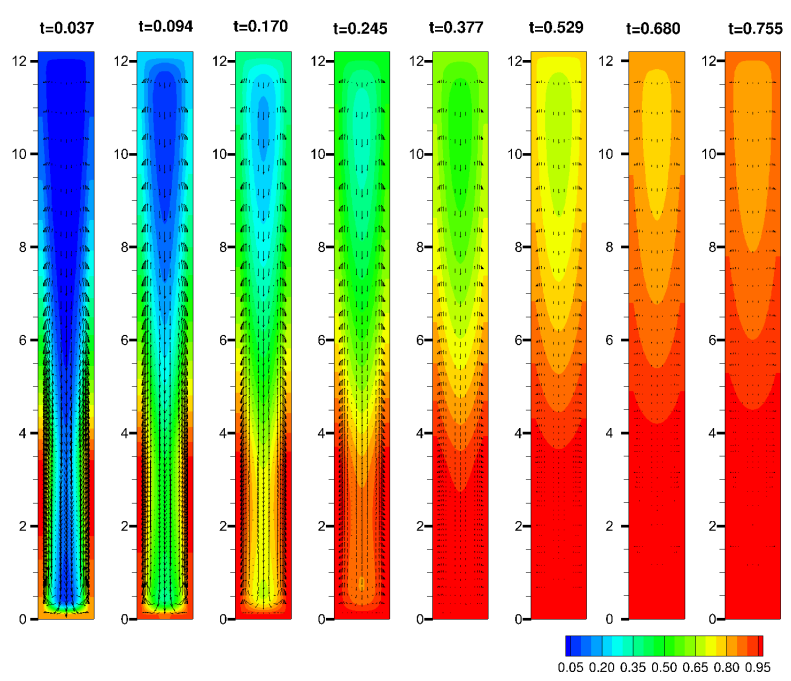

Fig. 2: Temporal evolution of the temperature field $R a=10^{4}$.

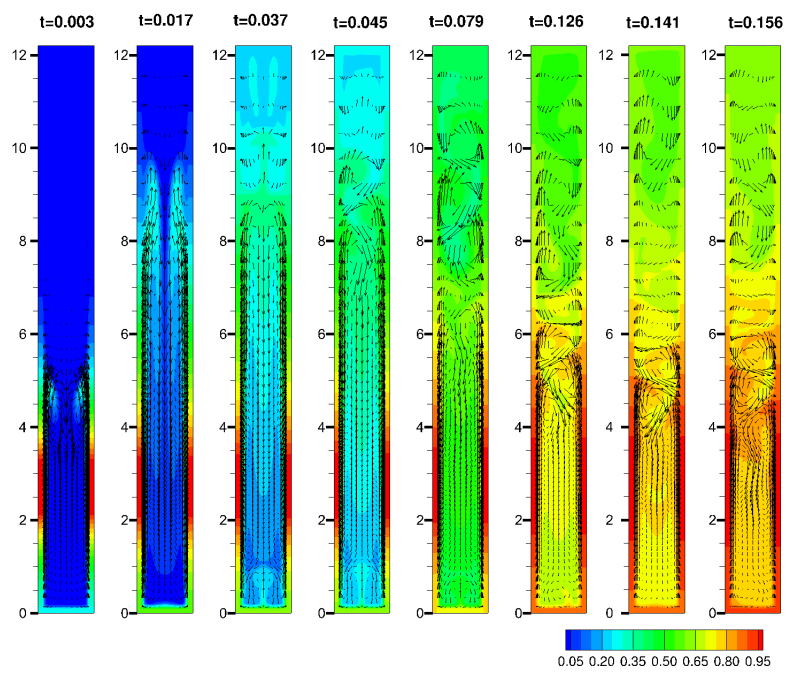

Fig. 3: Temporal evolution of the temperature field $R a=10^{6}$.

\section{Numerical Method}

The governing equations of the system were discretized under the control volumes scheme and solved by means of the SIMPLE algorithm (Patankar (1980)). Numerical codes in Fortran 90 language parallelized with the standard OpenMP (Open Multi Processing) were developed. The resulting system of algebraic equations are solved with the Tridiagonal Matrix Algorithm (TDMA), a line by line sweep and an iterative method.

Three different meshes, each one with 84 nodes in the horizontal direction and 184 nodes in the vertical one, were used according with the staggered grid scheme, with 10 nodes used for each solid wall. The meshes were generated by the coordinate transformation functions used by Martínez-Suástegui and Treviño (2008). The results obtained with these meshes were compared with those obtained with meshes with 180 nodes in the horizontal direction and 250 nodes in the vertical one for the largest value of the Rayleigh number studied, no significant variations were found.

A forward finite difference scheme was used to model the transient term with a time increment of $\Delta \tau=5 \times 10^{-4}$. Convergence for each time step was declared when the residual of each equation was less than $10^{-10}$.

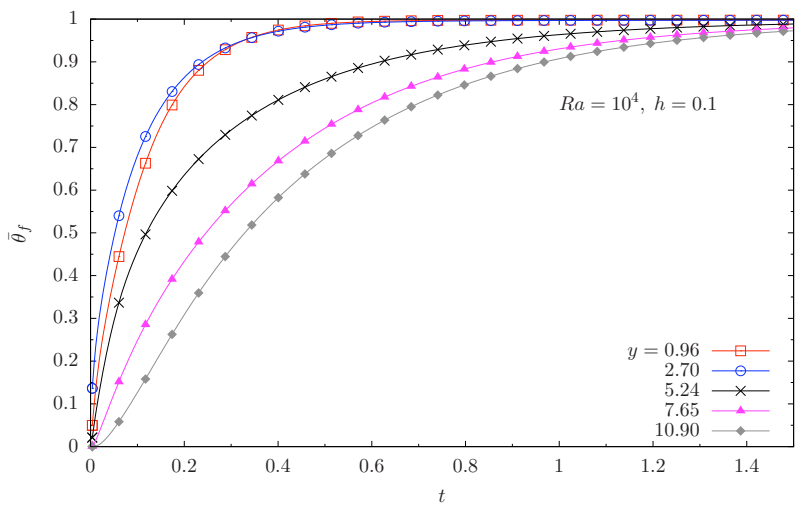

(a)

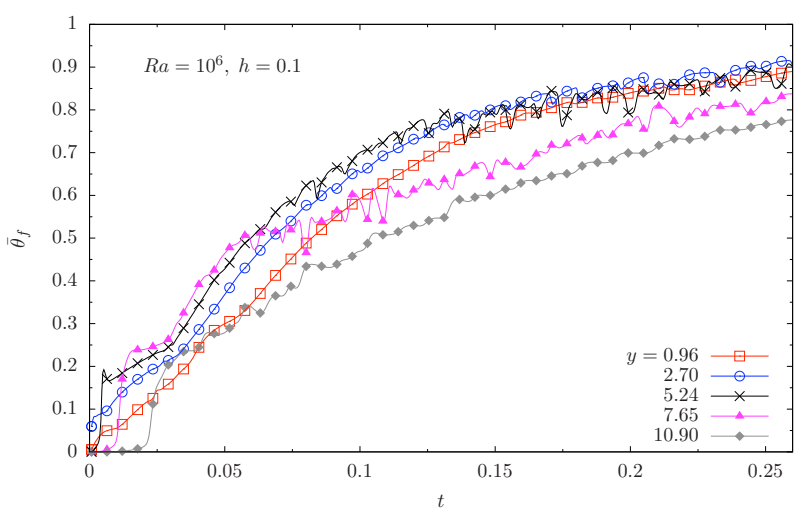

(b)

Fig. 4: Transverse averaged non-dimensional temperature in the fluid as a function of time for five different values of the vertical coordinate. $(a)$ $R a=10^{4}$, (b) $R a=10^{6}$.

\section{Results}

Figure 2 shows the evolution of the temperature field for the Rayleigh number, $R a=10^{4}$ and 3 shows the corresponding to $R a=10^{6}$. When $R a=10^{4}$, two symmetric recirculation regions 
develop in front of the heat sources whose height increases as the temperature increases on the walls. The shape of the isotherms indicates that the process is dominated by heat diffusion and is symmetric in all the studied time interval. In the case of $R a=10^{6}$, Fig. 3 shows that again two recirculation regions develop in front of the heated portions of the wall. However, convection is stronger than in the previous case, and the conjugated effects of convection and conduction on the walls promote the appearance of zones where thermal energy concentrates, giving place to new recirculation zones which are absent in the cases of smaller Rayleigh number. This leads the system to a unstable vortex configuration and to the symmetry break down in the end.

In Fig. 4, the transverse averaged non-dimensional temperature in the fluid is shown for five different values of the vertical coordinate, $\tilde{\theta}_{f}(y, t)=\int_{0}^{1} \theta_{f}(x, y, t) d x$. It can be observed that the thermal signal travels faster to the top of the cavity when the Rayleigh number increases and the overall temperature differences in the fluid decreases.

To analyze the vortex dynamics, the first moment of the transverse temperature distribution (thermal centroid) is calculated, $\tilde{x}(y, t)=\int_{0}^{1} x \theta_{f} d x / \tilde{\theta}_{f}(y, t)$, and is shown in the Fig. 5 for $R a=10^{6}$ and five different values of the vertical coordinate. The system loses symmetry in $y \approx 11$ at non-dimensional time of $t \approx 0.03$ showing an intense vortex shedding activity with different frequencies.

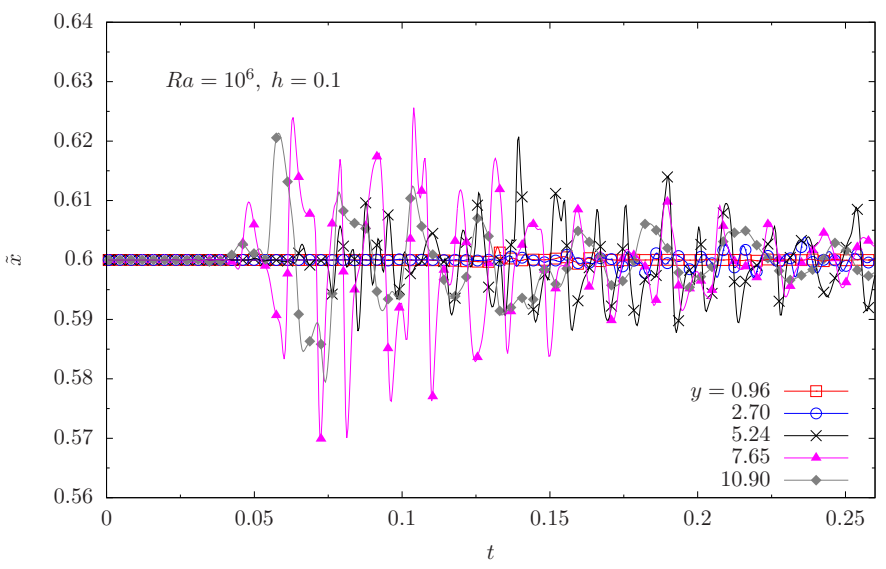

Fig. 5: Thermal centroid for $R a=10^{6}$ as a function of time. For a fixed value of $y$, positive values of $\tilde{x}(y, t)-0.6$ represent anticlockwise vortex.

The temporal evolution of the entropy generation field can be observed in Fig. 6 and 7. For the case of $R a=10^{4}$, it can be observed that the larger contributions to the entropy generation are localized in the solid-fluid interface and that the entropy generation is less intense inside the fluid, being practically null along the vertical symmetry axis of the cavity. In the case of the larger value of the Rayleigh number, $R a=10^{6}$, the entropy generation is more intense in general. As a consequence of the more intense convective heat flux, new entropy production zones appear inside the fluid and remarkably in mid zone of the base. When symmetry breaks down, the entropy production field show clearly the zones of more intense heat flux. For both cases, the entropy production decreases as time goes by, and the temperature becomes more uniform along the cavity.

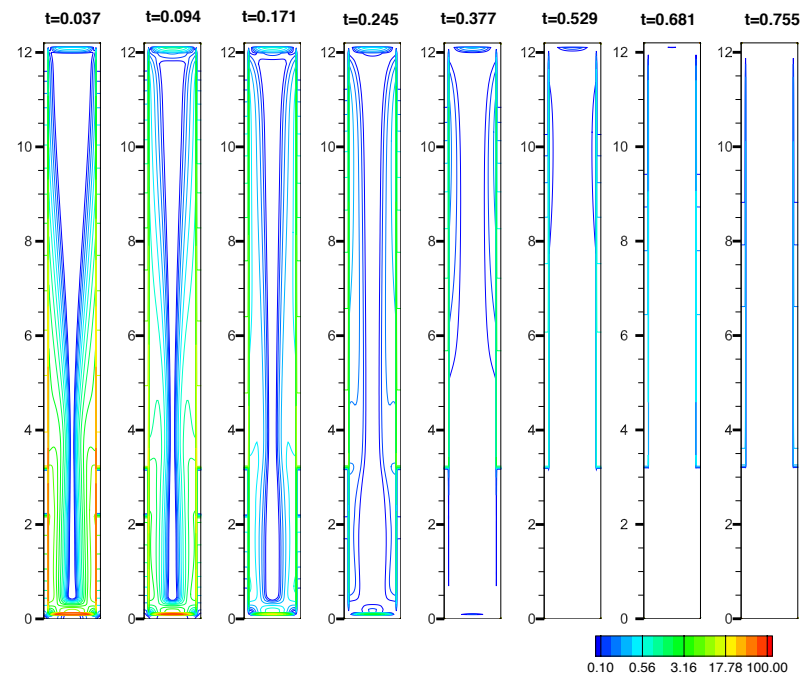

Fig. 6: Temporal evolution of the entropy generation field $R a=10^{4}$.

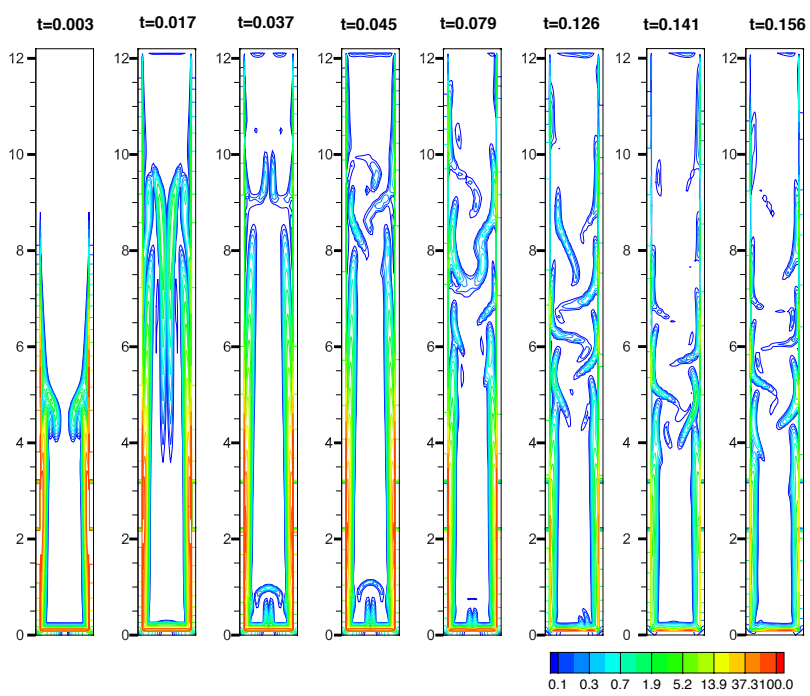

Fig. 7: Temporal evolution of the entropy generation field $R a=10^{6}$.

Figure 8 shows the average Nusselt number in both, the solid and the fluid around each one of the heat sources as a function of the non-dimensional convective time, $\tau=\sqrt{\operatorname{RaPr} t}$

$$
\bar{N} u_{f}(t)=\left.(-1)^{n} \int_{h+L_{1}}^{h+L_{1}+l} \frac{\partial \theta_{f}}{\partial x}\right|_{x=x_{n}} d y \quad \text { in the fluid, }
$$


where $n=1,2$ and makes reference to the heat sources located in the left and right side wall of the cavity respectively.

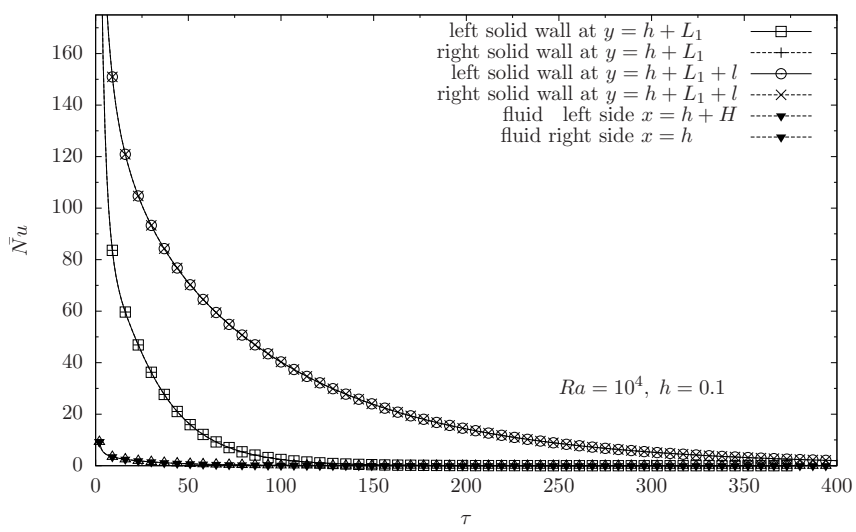

(a)

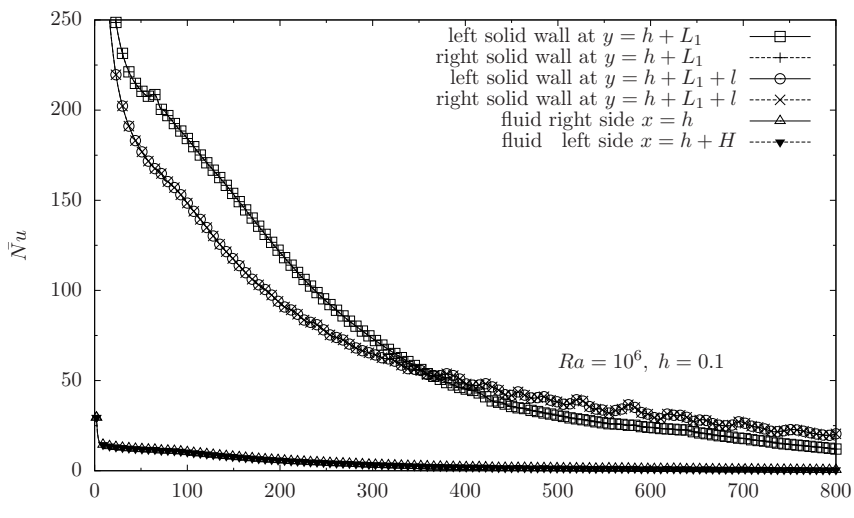

(b)

Fig. 8: Average Nusselt number as a function of the non-dimensional convective time in the solid and in the fluid around the heat sources (a) $R a=10^{4}$, (b) $R a=10^{6}$.

$$
\begin{aligned}
& \bar{N} u_{s}(t)=\left.K \frac{(-1)^{m}}{h} \int_{0}^{h} \frac{\partial \theta_{s}}{\partial y}\right|_{y=y_{m}} d x ; \\
& \bar{N} u_{s}(t)=\left.K \frac{(-1)^{m}}{h} \int_{h+H}^{2 h+H} \frac{\partial \theta_{s}}{\partial y}\right|_{y=y_{m}} d x
\end{aligned}
$$

in the left and right side of the solid respectively. In both expressions $m=1,2$ and indicates the bottom or the top of the heat source respectively.

In both cases the heat flux to the solid is larger than the heat flux to the fluid, this is so because of the large value of the thermal conductivities ratio $K$. When the buoyancy parameter increases, and after an initial period of non-dimensional time, the heat flux that travels across the solid wall in the upward direction decreases in relation to the one that travels across the solid wall in the downward direction. This effect is due to the increment in the speed of convective heat transfer that occurs for high values of the Rayleigh number. When the speed of convective heat transfer in the fluid is comparable to the rate of heat conduction on the solid, the temperature difference in the solid-fluid interface becomes small, thermal energy accumulates in the wall above the heat source and the temperature in this zone tends to be uniform. Thus, the heat flux represented by the Nusselt number decreases.

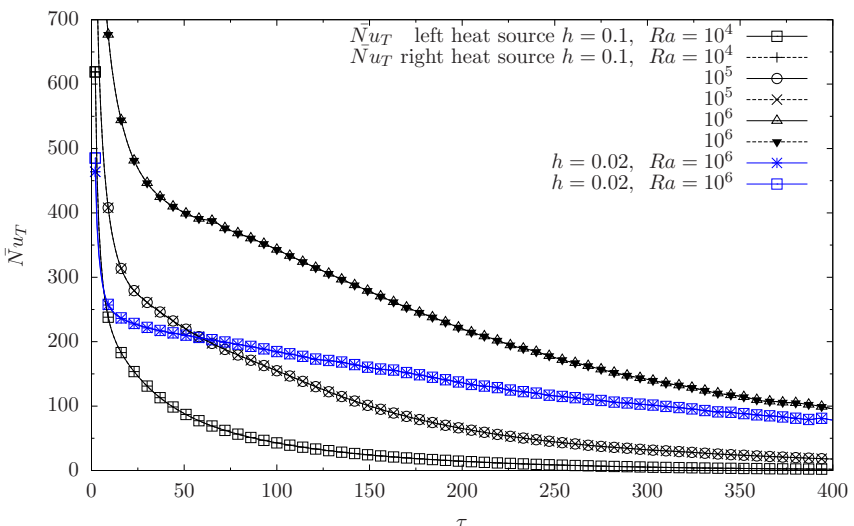

(a)

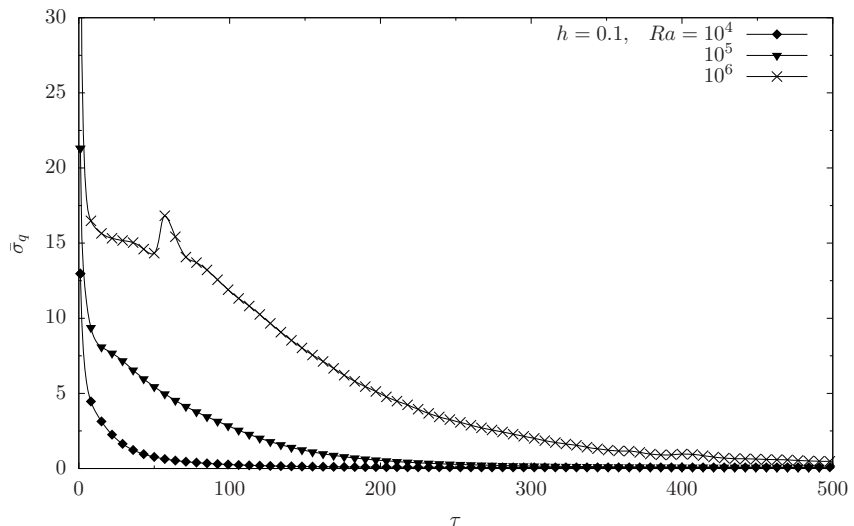

(b)

Fig. 9: (a) Average total Nusselt number as a function of the nondimensional convective time for $R a=10^{4}, 10^{5}$ and $10^{6}$. (b) Total Entropy production as a function of the non-dimensional convective time for $R a=10^{4}, 10^{5}$ and $10^{6}$.

In the Fig. 9(a) the sum of the three contributions to the average Nusselt number is plotted as a function of the non-dimensional convective time. From the curves corresponding to $h=0.1$ and the three different values of the Rayleigh number, it is clear that the overall heat flux to the system increases as the Rayleigh number increases. Meanwhile, from the curves corresponding to $R a=10^{6}$ with $h=0.1$ and $h=0.02$ it can be observed that the overall heat flux decreases with the thickness of the wall. 


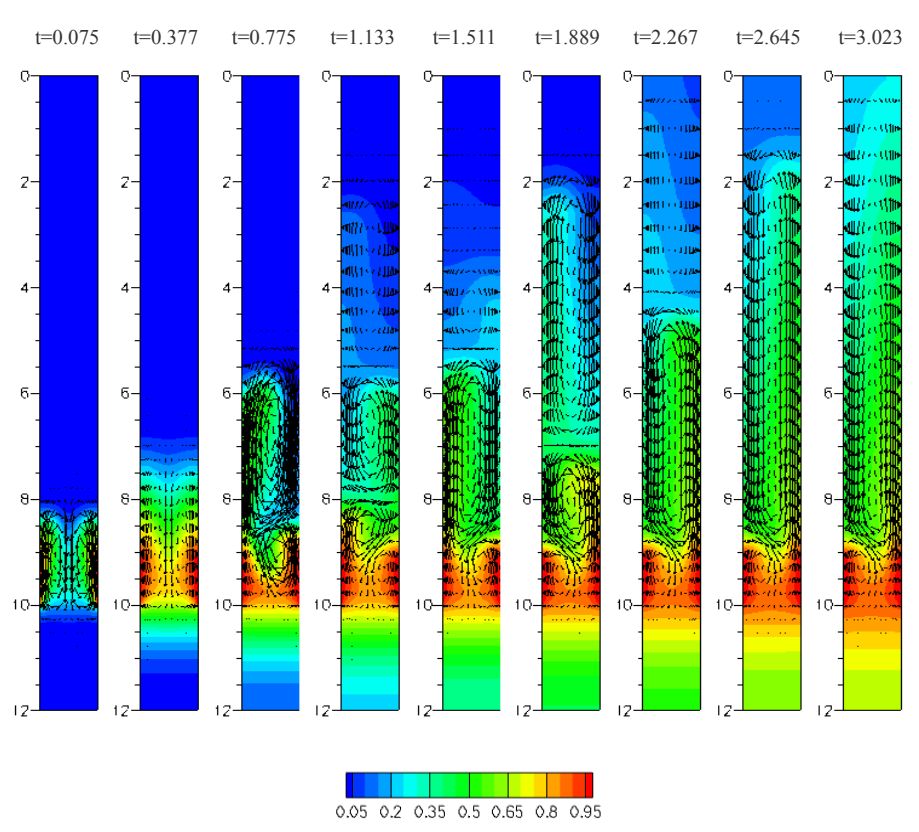

Fig. 10: Temporal evolution of the temperature field $R a=10^{4}$ for adiabatic walls.

The Fig. 9(b) shows the overall entropy production as a function of the non-dimensional convective time $\tau$, and illustrates the direct influence of the increment of the buoyancy forces on the increment of the total entropy production of the system. The curves describe a similar behavior to that followed by the curves of the Nusselt number due to the direct relation that exist between this two quantities. It can be observed as well that when the buoyancy forces are large, the total entropy production increases, however the average temperature in the cavity increases faster in this case. This suggest that a good equilibrium between time of heating and entropy production can be achieved for a practical purpose with little more investigation.

For comparison, the case corresponding to $R a=10^{4}$ and adiabatic walls is included in Fig. 10. Two vortical structures develop above the heated plates displacing the warmer fluid to higher regions of the cavity close to its walls while the colder fluid moves to lower regions of the cavity along the symmetry plane. The height of the upper heated layer increases with time with a sinusoidal shape until instability occurs, resembling to that of Rayleigh-Bénard problem. This contrasts with the smooth behavior for the case showed in Fig. 2 for the same Rayleigh number but with a heat conductive wall where no spontaneous instability occurs. Similar results are obtained for the higher values of the Rayleigh number, the process with the heat conductive wall is much faster and smoother than the obtained with adiabatic walls.

\section{Conclusions}

Conjugated conduction-natural convection, in a rectangular cavity with large aspect ratio and solid conductive walls of finite thickness, was numerically studied with the use of the control volume discretization method and the SIMPLE algorithm. Isotherms, entropy production fields, heat flux to the system given by the average Nusselt number and a quantitative information of the vortex dynamics were obtained. Different mechanisms of symmetry break down and heat transfer inside the fluid were found depending on the Rayleigh number. It was possible to show that an increment in the Rayleigh number provokes an increment in the heat flux over the solid walls and inside the fluid, making a faster process of heat transfer that produces more entropy. It was observed that the overall heat flux decreases with the thickness of the wall for the considered values of $h$. Comparing the present results with a previous analysis of the same system but with adiabatic walls, it can be observed that the presence of the solid conductive walls rises the speed of heating and tends to stabilize the process.

\section{References}

Kim, D.M., Viskanta, R.. Effect of wall heat conduction on natural convection heat transfer in a square enclosure. Journal of Heat Transfer 1985;107(1):139-146.

Liaqat, A., Baytas, A.C.. Conjugate natural convection in a square enclosure containing volumetric sources. International Journal of Heat and Mass Transfer 2001;44:3273-3280.

Martínez-Suástegui, L., Treviño, C.. Transient laminar opposing mixed convection in a differentially and asymmetrically heated vertical channel of finite length. Heat Mass Transfer 2008;51:5991-6005.

Moghtada, , Mobedi, . Conjugate natural convection in a square cavity with finite thickness horizontal walls. International Communications in Heat and Mass Transfer 2008;35(4):503 - 513.

Patankar, S.V.. Numerical heat transfer and fluid flow. Hemisphere Publishing Corporation, 1980.

Varol, Y., Oztop, H.F., Koca, A.. Entropy generation due to conjugate natural convection in enclosures bounded by vertical solid walls with different thicknesses. International Communications in Heat and Mass Transfer 2008;35(5):648 - 656.

Varol, Y., Oztop, H.F., Pop, I.. Entropy analysis due to conjugatebuoyant flow in a right-angle trapezoidal enclosure filled with a porous medium bounded by a solid vertical wall. International Journal of Thermal Sciences 2009;48(6):1161 - 1175.

Zhang, W., Zhang, C., Xi, G.. Conjugate conduction-natural convection in an enclosure with time-periodic sidewall temperature and inclination. International Journal of Heat and Fluid Flow 2011;32(1):52 - 64 . 


\section{Response to reviewer 1.} Comments:

please specify the specific application of this topic.

A study like the present can be useful in the design of low energy water heating devices.

\section{Corrections:}

incorporate the assumptions in section 2 before writing the governing equations.

The assumptions concerning the properties of the fluid and the solid were incorporated in section 2.

\section{Response to reviewer 2.}

Comments:

On what basis was the size of the mesh determined? There must be a mesh convergence study to support the choice.

A mesh convergence study was performed for meshes of $84 \times 184,180 \times 250$ nodes for the case of $R a=10^{6}$ with walls of thickness 0.1. The results for the total averaged Nusselt number are shown in Fig. 11. No significant variations were found.

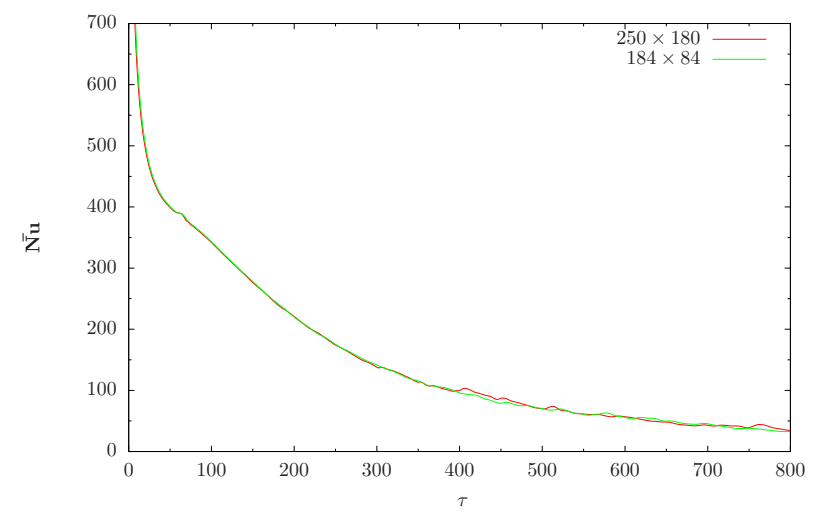

Fig. 11: Comparison between different mesh sizes for $R a=10^{6}$.

How can the authors be sure that the vortex shedding discussed in the paragraph following Figure 4 is not initiated by the accumulation of numerical errors. Did they check the effect of how the transient terms were being approximated? There needs to be a statement regarding what methods were used to advance through time. The SIMPLE algorithm is rather old fashioned in this respect. How many iterations were used at each time step?

The code was tested for different mesh sizes and time increments for the case of adiabatic walls and Rayleigh number of $10^{6}$. The two lower values of the non-dimensional time increment tested were $\Delta \tau=5 \times 10^{-4}$ and $\Delta \tau=10^{-4}$. The vortex activity found in this case is more intense than that found in the cases where the conductive walls are present. Again, no significant variations on the results were found. Specially, the symmetry break down and the vortex shedding that follow were independent of the time increment used. Thus, the authors are sure that the physical onset is unstable, this unstable configuration is the one that amplifies the differences that exist in the numerical solution and promote the symmetry break down and not the mere accumulation of numerical errors. A similar study is now being performed for the case of $R a=10^{6}$ with the conductive wall of thickness 0.1 .

A statement concerning the non-dimensional time increment and the number of iterations has been included in section 3 .

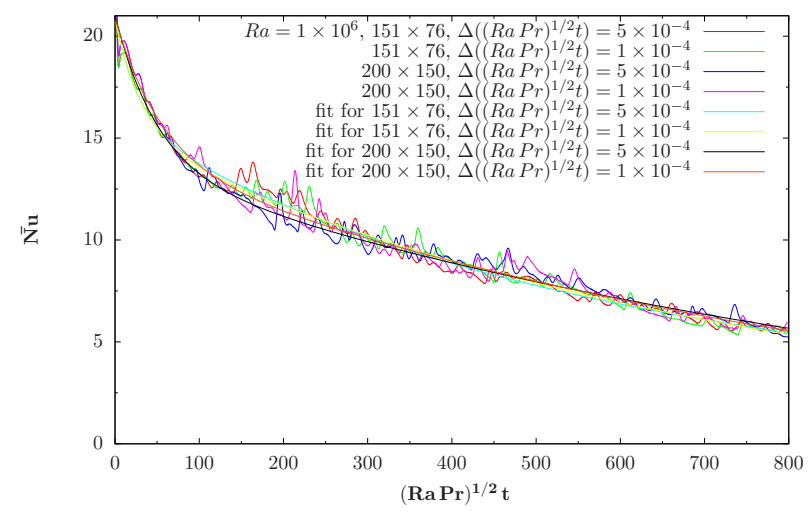

Fig. 12: Comparison between the results for the average Nusselt number for different grid size and different time increments in the case of $R a=10^{6}$ for adiabatic walls.

The problem studied is self stabilizing in that eventually the fluid heats up to the temperature of the plates and all buoyancy disappears? The authors should comment on the relevance of the study to a physical situation.

A physical situation of this kind is found in a water heater, this study can be relevant in the design of such devices.

\section{Corrections:}

Figures 2 and 5 should be bigger or rearranged to make the visualizations clearer. The numbers are very hard to read.

The figures size has been changed to make it easier to read.

The last sentence in the conclusion should be omitted because there is no supporting evidence in the body of the paper.

Figure 10 has been added in order to support the last sentence in the conclusions. 
The following minor corrections should be made.

Last line in abstract: should read 'fixed values for the rest of the parameters'.

End of 3rd paragraph in introduction should be 'obtaining an energy saving'.

2nd line in Section 2. It should be 'Boussinesq' not 'Boussinesqâs'.

Near the end of the first paragraph in Section 4, 'apparition' should be replaced by 'appearance' (apparition can mean 'a remarkable or unexpected appearance' â which is a bit strong in this instance.)

End of first paragraph in Section 4, the word 'break' should be replaced by 'break down'. (A similar change should be made in the middle of the conclusions.)
Paragraph after equation (8). It is unusual to talk about the speed of conductive heat transfer. 'rate of head conduction' would be better.

Minor corrections have been taken into account.

\section{Comment to reviewers.}

In order to provide some information about the dependence of the averaged Nusselt number on the thickness of the wall, the Fig. 9 was modified and the result for $R a=10^{6}$ with $h=0.02$ was included. Further calculations are being performed to extend the present data. 